\title{
Morphology Control of Novel Cross-Linked Ferrocenedimethanol Derivative Cyclophosphazenes: From Microspheres to Nanotubes and Their Enhanced Physicochemical Performances
}

Yasir Abbas ${ }^{\dagger}$, ZareenZuhra ${ }^{\dagger}$, Majid Basharat ${ }^{\dagger}$, MunanQiu ${ }^{\dagger}$, Zhanpeng $\mathrm{Wu}^{\dagger, *}$, Dezhen $\mathrm{Wu}^{\ddagger}{ }^{\ddagger},{ }^{*}$, Shafqat Ali ${ }^{* *}$

$\dagger$ Key Laboratory of Carbon Fiber and Functional Polymers (Beijing University of Chemical Technology), Ministry of Education, Beijing, 100029, China

IState Key Laboratory of Chemical Resource Engineering,Institute of Science, Beijing University of Chemical Technology, Beijing 100029, China

*The Key Laboratory of Advanced Materials of Ministry of Education, School of Material Science and Engineering, Tsinghua University, Beijing 100084, China

*To whom correspondence should be addressed. Email: wuzp@mail.buct.edu.cn (Z.Wu).Email: wdz@mail.buct.edu.cn(D.Wu).Email:shafqatali@mail.tsinghua.edu.cn (S. Ali)

\section{Figure Captions}

Figure S1. SEM image revealing mixed morphology of nanotubes and microspheres

Figure S2. SEM images of the cross-linked Poly(ferrocenedimethano)cyclotriphosphzene nanotubes (a) and microspheres (b) when the concentrations of HCCP were 0.5 and 1,1'ferrocenedimethanol concentration was 7.0 Mm. 
Figure S3. EDX map of the cross-linked Poly(ferrocenedimethano)cyclotriphosphzene nanotubes.

Figure S4. $\quad$ FT-IR spectra of oxidized cross-linked Poly(ferrocenedimethano)cyclotriphosphzenenanotubes and microspheres.

Figure S5. SEM images of the cross-linked Poly(ferrocenedimethano)cyclotriphosphzenenanotube (a) and microspheres (b) after oxidation with tetracyanoethylene.

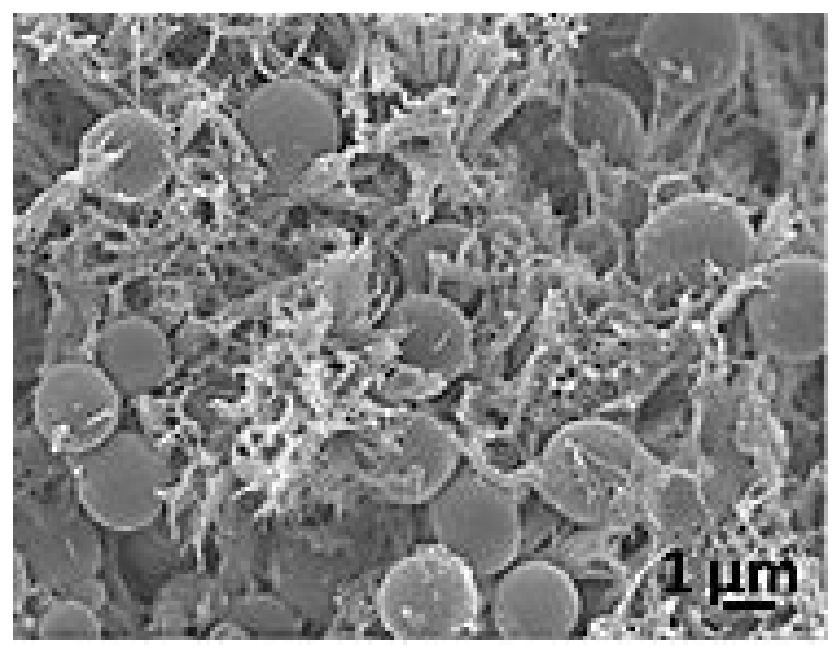

Figure S1. SEM image revealing mixed morphology of nanotubes and microspheres. 

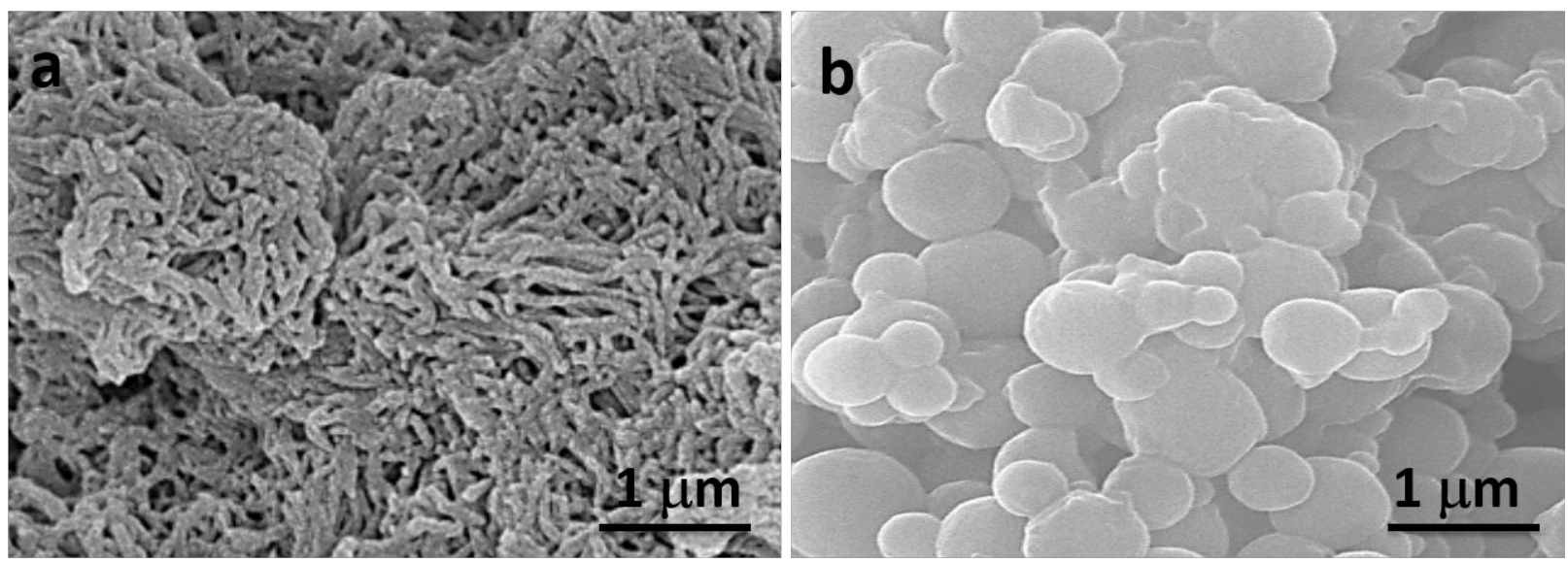

Figure S2. SEM images of the cross-linked Poly(ferrocenedimethano)cyclotriphosphzene nanotubes (a) and microspheres (b) when the concentrations of HCCP were 0.5 and 1,1'ferrocenedimethanol concentration was $7.0 \mathrm{mM}$.
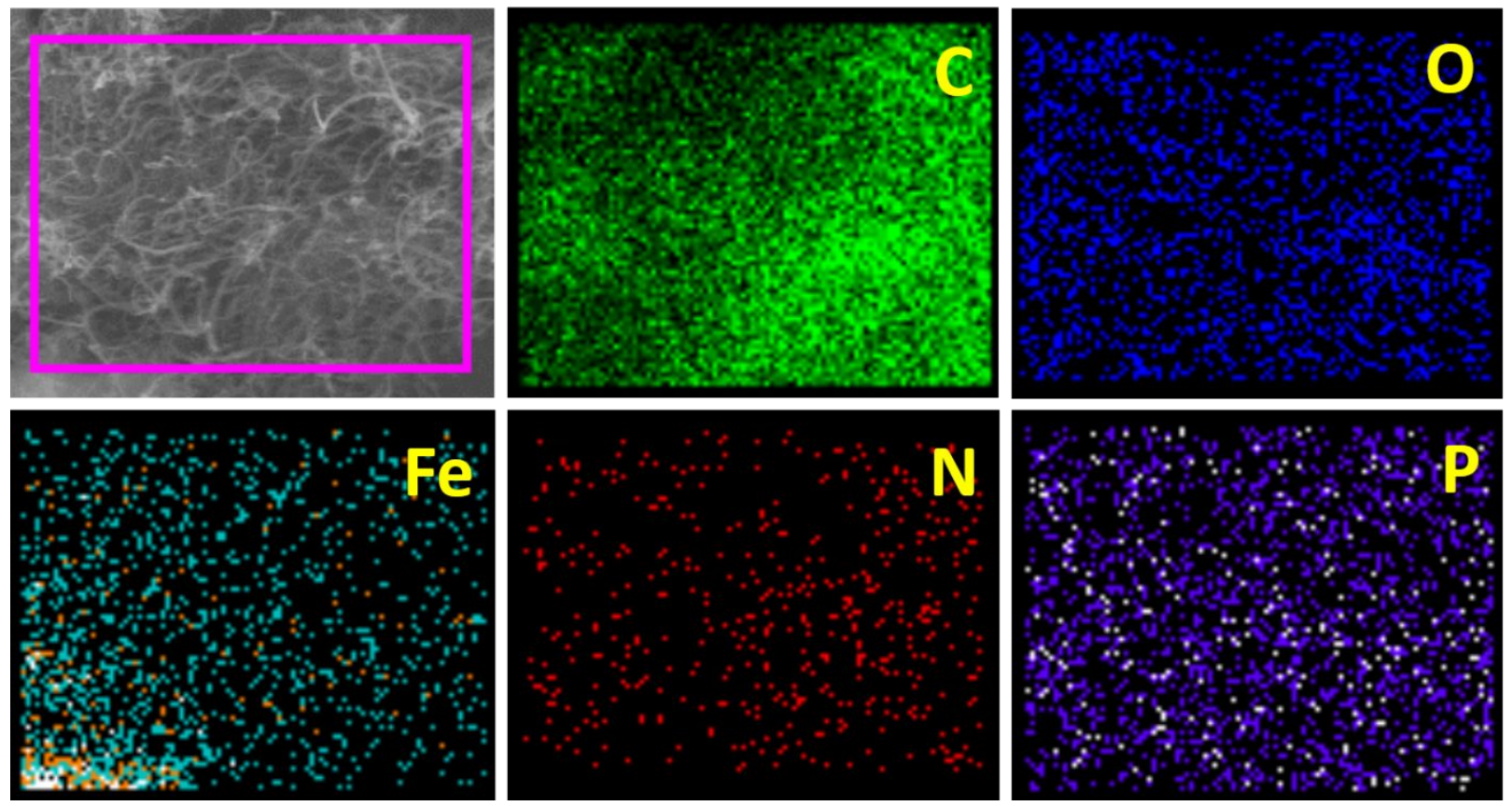
Figure S3. EDX map of the cross-linked Poly(ferrocenedimethano)cyclotriphosphzene nanotubes.

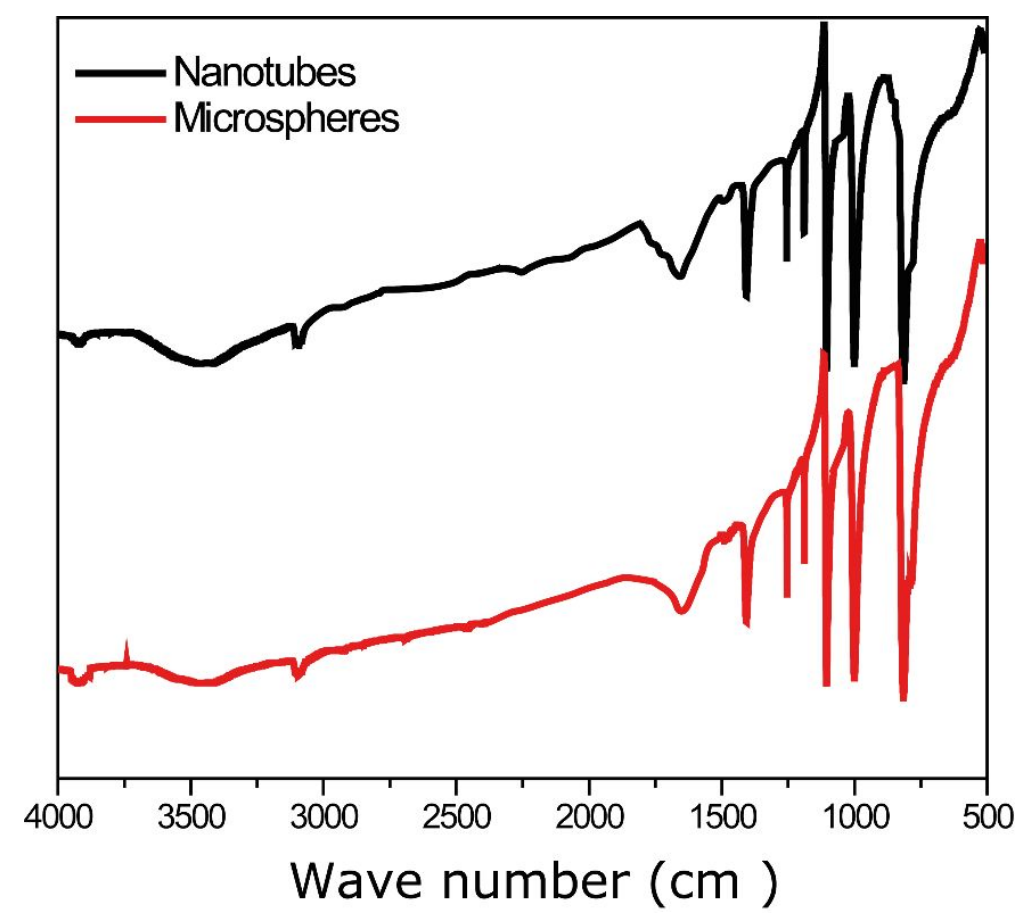

FigureS4. FT-IR spectra ofoxidizedcross-linked

Poly(ferrocenedimethano)cyclotriphosphzenenanotubes and microspheres.
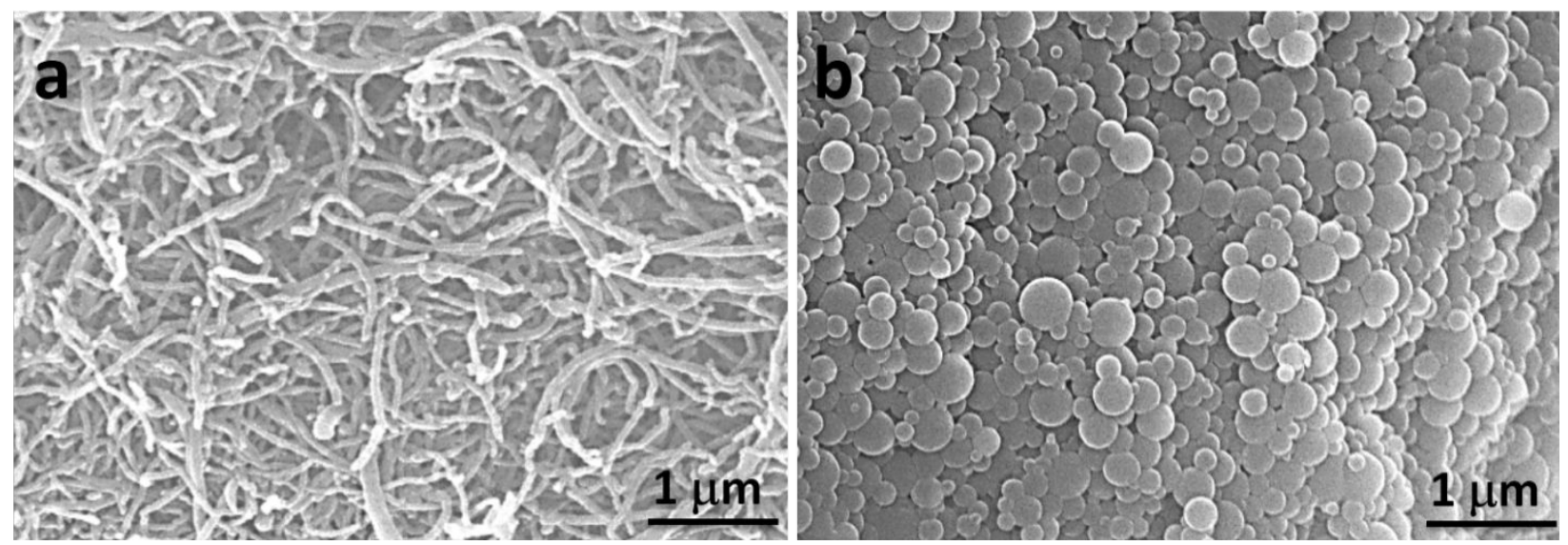
Figure S5. SEM of the cross-linked Poly(ferrocenedimethano)cyclotriphosphzenenanotube (a) and microspheres (b) after oxidation with tetracyanoethylene. 